\title{
ANÁLISIS CRÍTICO DE DISCURSO DEL BIENESTAR SUBJETIVO Y LA PSICOLOGÍA POSITIVA EN LA ADOLESCENCIA
}

\author{
CRITICAL DISCOURSE ANALYSIS OF SUBJECTIVE WELL-BEING AND POSITIVE \\ PSYCHOLOGY IN ADOLESCENCE
}

\author{
Daniela Fernández Olguín* \\ Universidad Viña del Mar, \\ Viña del Mar - Chile. \\ Recibido abril de 2018/Received April, 2018 \\ Aceptado noviembre de 2018/Accepted November, 2018
}

\begin{abstract}
RESUMEN
El siguiente artículo desarrolla un análisis crítico de discurso del bienestar subjetivo y la psicología positiva en la adolescencia, a partir de la revisión bibliográfica de investigaciones recientes sobre estos temas, planteando que el bienestar subjetivo en los adolescentes implica una categorización y homogeneización respecto a ciertos ideales de carácter político que buscan orientar las acciones de estos sujetos en relación a sí mismos, dentro de una lógica neoliberal. Finalmente, se propone el re-pensar la relación que se ha establecido entre bienestar subjetivo, psicología positiva y adolescencia, dando cuenta de sus implicancias políticas.

Palabras Clave: Bienestar subjetivo, Psicología Positiva, Adolescencia, Gubernamentalidad, Psicopolítica.
\end{abstract}

\begin{abstract}
The following paper develops a critical discourse analysis of positive psychology and subjective well-being recent researches in adolescence, arguing that the notion of subjective well-being for adolescents implies a categorization and homogenization when it comes to certain political ideals, in order to guide some actions that these subjects make regarding themselves, within a neoliberal logic. With that being said, this article proposes the re-thinking of the established relation between subjective wellbeing, positive psychology and adolescence, showing their political implications.
\end{abstract}

Key Words: Subjective Well-Being, Positive Psychology, Adolescence, Governmentality, Psychopolitics.

\section{Definición y antecedentes sobre el concepto de bienestar subjetivo}

El estudio del bienestar subjetivo se ha posicionado como un área de investigación prolífica dentro de la psicología. Según plantea Vásquez y Chavez (2016), históricamente la psicología se había encargado de estudiar al individuo desde la perspectiva del déficit, es decir, poniendo mayor énfasis en la enfermedad y no en aspectos positivos de la vida. Los estudios sobre el bienestar subjetivo mayormente han sido propuestos por la Psicología Positiva, cuyo objetivo es poner su foco y estudiar

* Autor correspondiente / Correspondig author: daniela.paz.f@gmail.com 
las fortalezas, virtudes, las emociones positivas, el bienestar subjetivo, entre otras áreas, así como los efectos de estas en las personas y sociedades, con el fin de facilitar su desarrollo y promover intervenciones vinculadas a mejorar la calidad de vida (Diener, 2000; Seligman \& Csikszentmihalyi, 2000; Seligman, 2005; Swartz, 2018). Además, la Psicología Positiva “(...) parte del concepto de un ser humano libre, prudente, racional y responsable de sus actos y sus consecuencias" (Fernández, 2008, p.165), poniendo énfasis en la responsabilidad del individuo por sus acciones y como agente de su historia.

Seligman (2011) manifestó la importancia del estudio de factores que tengan por objetivo mejorar la calidad de la vida y prevenir los trastornos de carácter psicológico, con el fin de lograr terapias de mayor éxito. A partir de este desarrollo, el bienestar subjetivo se puede definir como "la percepción y experiencia personal de respuestas positivas y negativas y las evaluaciones específicamente cognitivas de la satisfacción con la vida" (Proctor, 2013, p.6437). Este término se introduce como una manera de identificar un campo de la psicología que buscara entender las evaluaciones de las personas sobre su calidad de vida y usualmente se intercambia con el término de felicidad (Proctor, 2013).

La Psicología Positiva ha mantenido una estrecha relación con el surgimiento del concepto de bienestar subjetivo, el cual se ha homologado con el término felicidad, si bien bienestar subjetivo implica una mayor operacionalización del concepto. El término bienestar subjetivo busca dar cuenta de la particularidad del sujeto en las variables que han medido históricamente el bienestar y la calidad de vida (Goodman, Disabato, Kashdan, \& Barry, 2017). Además, el sujeto es quien debe -mediante ciertos parámetros e indicadores expuestos mayormente desde el método científico- agenciar su bienestar subjetivo, mediante sus recursos, fortalezas y virtudes.

Según García (2002), las definiciones que han sido construidas en torno a la noción de bienestar subjetivo pueden ser agrupadas en tres categorías: una primera en la cual las descripciones sobre el bienestar realizadas por el sujeto se relacionan con la satisfacción vital. Una segunda categoría, en la cual es posible observar la definición de bienestar relacionada con la predominancia de los afectos positivos sobre los negativos en la valoración del propio sujeto. Y finalmente, una última categoría en la cual se propone que la felicidad es una virtud y un valor en sí mismo. Así, el bienestar subjetivo es un concepto multidimensional que integra distintas variables que el sujeto debe integrar y alcanzar, mediante sus propias valoraciones y recursos.

El concepto de bienestar subjetivo apela a "un área general de interés científico y no una estructura específica que incluye las respuestas emocionales de las personas, satisfacciones de dominio y los juicios globales de satisfacción de la vida" (Cuadra \& Florenzano, 2003, p. 85). Este concepto se encuentra también en relación con el de "bienestar psicológico", el cual alude a una perspectiva cercana a la teoría humanista, donde se ponen en juego conceptos como autorrealización, potencial humano y plenitud (Alfaro, Casas, \& López, 2015). Las investigaciones desarrolladas respecto al concepto de bienestar subjetivo se enfocan sobre todo en la evaluación cuantitativa de intervenciones que buscan el desarrollo del bienestar subjetivo de los sujetos en distintos contextos (Arps, Friesen, \& Overall, 2018; Ryff, 2018; Russell \& Daniels, 2018).

Esto se ha desarrollado en temáticas como las adicciones (Ghahari, Farhangui, \& Gheytarani, 2017; Khazaei, Khazaei, \& Ghanbari-H, 2017), la psicología clínica aplicada (Antoine, Dauveir, Andreotti, \& Congard, 2018), las intervenciones en trastornos alimenticios y de la imagen corporal (Tylka, 2012; Flink, Smeets, Bergbom, \& Peters, 2015) y la depresión (Walsh, Szymczynka, Taylor, \& Priebe, 2018), entre otras. Además, conceptos vinculados a la psicología positiva y bienestar subjetivo han sido trabajados en otras áreas, destacando por ejemplo el ámbito organizacional (Muha \& Manion, 2010; Lyngdoh, Liu, \& Sridhar, 2018) y educacional (Voerman, Korthagen, Meijer, \& Simons, 2014; Burkhardt, Manicavasagar, Batterham, \& Hadzi-Pavlovic, 2016).

A pesar de este desarrollo teórico e investigativo, surgen algunos cuestionamientos en torno al desarrollo e implicancias de este modelo teórico, considerando sus fundamentos, así como sus implicancias en la concepción de sujeto (PrietoUrsúa, 2006; Christopher \& Hickinbottom, 2008; Fernández, 2008; Miller, 2008). En base a esto, se torna relevante poder problematizar y revisar los supuestos e implicancias de la psicología positiva en sus investigaciones y posicionamientos, y, específicamente, reconociendo las particularidades 
del concepto bienestar subjetivo propuesto por la psicologia positiva en las investigaciones realizadas sobre la adolescencia.

Para esto, se realizará un Analisis Crítico del Discurso (Fairclough, 1989, 1995) de veintitres investigaciones publicadas (en inglés y español) entre los años 2010 y 2018, respecto a las temáticas de adolescencia, bienestar subjetivo y psicología positiva. Además, se revisaron libros e investigaciones publicadas vinculados al tema de bienestar subjetivo y psicología positiva general (también en inglés y español). El Análisis Crítico del Discurso ve el uso del lenguaje como una forma de práctica social, en donde se reproducen relaciones de poder, ideologías y discursos hegemónicos (Fairclough, 1992; Janks, 2006). En base a esto, se analizaron los textos a partir de las dimensiones de análisis propuestas por Fairclough $(1989,1995)$ : el análisis descriptivo del texto, los procedimientos de análisis interpretativo y el análisis social explicativo.

\section{Adolescencia y bienestar subjetivo}

En los estudios sobre la adolescencia ha existido una tendencia hacia la indagación de este período desde un discurso biomédico, bajo la predominancia de un enfoque biologicista para la comprensión de los cambios y advenimientos que este período conlleva (Cornejo, 2015).

Ante la complejidad de esta etapa, el concepto de bienestar subjetivo surge como un campo particular de estudio: "el adolescente debe atravesar numerosas situaciones de cambio con relación a sí mismo y al entorno, lo que incide en su autoapreciación y en el empleo de recursos para enfrentar las nuevas circunstancias desde lo intrapsíquico y lo interpersonal" (Álvarez, 2014, s/p). De este modo, pareciera circunscribirse a la adolescencia como una etapa que invoca potenciales peligros debido a la fragilidad e incertidumbre de los y las adolescentes en su proceso identitario, y en el cual las medidas protectoras serían necesarias con el fin de favorecer el bienestar subjetivo de este grupo, que se integra al mundo adulto.

A partir de esto, es posible observar diferentes lineamientos que las investigaciones sobre bienestar subjetivo desde la psicología positiva en la adolescencia siguen. Casas, FernándezArtamendi, Monserrat, Bravo, Bertrán y del Valle (2013) refieren la focalización de estos estudios en el área cuantitativa. Por ejemplo, se encuentran las investigaciones de Coronel (2010), quien refiere la indagación del bienestar en la adolescencia mediante escalas cuantitativas que buscan identificar la presencia o ausencia de emociones positivas y negativas, a su vez de la relación con ciertos rasgos de la personalidad asociados a la normalidad o no. En el marco de esta investigación, la escala BIEPS-J (Escala de Bienestar Psicológico para Adolescentes), se posiciona como un instrumento cuantitativo y objetivo para investigar sobre la percepción de los adolescentes respecto a su bienestar.

Por otro lado, Castellá, Saforcada, Tonon, Rodríguez de la Vega, Mozobancyk y Bedin (2012) realizan un estudio comparativo de carácter mixto respecto al bienestar subjetivo de adolescentes de Argentina y Brasil. Ortuño-Sierra, Aritio-Solana, Chocarro de Luis, Navaridas y Fonseca-Pedrero (2017) exponen las propiedades psicométricas de la Escala de Satisfacción con la Vida en una muestra de adolescentes españoles, como instrumento útil para conocer la satisfacción con la vida con auto reportes de los adolescentes. Es importante precisar que, a pesar de la prevalencia de estudios de carácter cuantitativo en esta temática, también se evidencian -en menor medida- estudios de carácter cualitativo, los cuales mantienen la misma perspectiva teórica y utilizan mayormente las entrevistas como instrumento de producción de información (González-Carrasco, Vaqué, Malo, Crous, Casas, \& Figuer, 2014; Favotto, Michaelson y Davidson, 2017; Lögdberg, Nilsson, \& Kostenius, 2018).

Dentro de las investigaciones también destacan estudios que dan cuenta de una visión más bien causal respecto al bienestar subjetivo y su relación con otras variables como la construcción de expectativas positivas a futuro (Eryilmaz, 2011), o a los factores sociales e interpersonales, destacando el rol de la familia (Lampropoulou, 2018; Bastaits, Pasteels, \& Mortelmans, 2018), así como la escuela o el grupo de pares (Sachs, Gillham, DeMaria, Andrew, Peabody, \& Leventhal, 2015; Van Outtsel, Ponnet, \& Walrave, 2017). Si bien es relevante el analizar las expectativas, así como la participación de la familia y comunidad escolar y social en la vida de los adolescentes, circunscribir esta relación a la asociación de variables, restringe las posibilidades de análisis e integración de la complejidad propia de estas relaciones en la adolescencia en el contexto actual. La importancia otorgada a los fundamentos científicos para sustentar estudios en torno al bienestar subjetivo plantea 
incluso investigaciones que relacionan los afectos positivos con la herencia genética (Cloninger \& García, 2015).

Finalmente, es relevante destacar cómo algunas de estas investigaciones focalizan su interés en aspectos individuales de los y las adolescentes para evaluar y proyectar el desarrollo de su bienestar subjetivo, como por ejemplo el rol de la gratitud en el comportamiento antisocial y prosocial de adolescentes (Bono, Froh, Disabato, Blalock, McKnight, \& Bausert, 2017) o la concordancia entre valores y comportamiento (Warren, Wray-Lake, \& Syvertsen, 2018). Destaca también la posibilidad de predecir a través de fortalezas de carácter el bienestar subjetivo del adolescente: "Investigaciones previas indican que varias fortalezas de carácter (por ejemplo, gratitud, optimismo, persistencia y autorregulación) se correlacionan positivamente con medidas de bienestar subjetivo en adolescentes" (Gillham, Adams-Deutsch, Werner, Reivich, Coulter-Heindl, Linkins, Winder, Peterson, Park, Abenavoli, Contero, \& Seligman; 2010, p.31).

Así, a partir de esta breve revisión en torno a las líneas de investigación que emergen como relevantes en torno al bienestar subjetivo en la adolescencia, surgen ciertos elementos importantes a analizar respecto al concepto de bienestar subjetivo, la psicología positiva y las posibles implicancias de estos discursos en el estudio de la adolescencia.

Surge de este modo la pregunta en torno a los discursos con los cuales se evalúan conductas adolescentes y cómo a su vez estos discursos construyen criterios e indicadores objetivos que dan cuenta de la medición del bienestar subjetivo o que inciden en su disminución. El sujeto adolescente como tal no es una categoría natural, sino que es resultado del desarrollo de fuerzas productivas en Occidente, como consecuencia de la necesidad detectada de una mayor preparación por parte de la sociedad burguesa a los individuos jóvenes, para así facilitar su mejor integración a la vida productiva y social, en una sociedad que se distingue diametralmente de la lógica de la sociedad feudal. Las transformaciones de una sociedad feudal hacia el capitalismo y neoliberalismo, inciden en la producción de subjetividades respecto a las necesidades del mercado para sustentar estos modelos económicos y políticos, desechando las anteriores pautas de trabajo, producción e instituciones (Le Breton, 2012).
Tal transformación conlleva ciertos cambios en las racionalidades de la gubernamentalidad, las cuales se posicionan en el contexto político y económico del neoliberalismo, sin el cual no sería posible comprender la emergencia del nuevo sujeto adolescente, categoría que dista de ser natural: "La sociedad regulada según el mercado en la que piensan los neoliberales es una sociedad en la cual el principio regulador no debe ser tanto el intercambio de mercancías como los mecanismos de competencia" (Foucault, 1979/2008, p.182). Así, el Estado debe responder a una lógica que permita las condiciones de la competencia.

Con la introducción del modelo del neoliberalismo norteamericano se individualiza el modelo de una empresa desde la sociedad al propio sujeto, a través del llamado sujeto emprendedor (Foucault, 1979/2008), en el cual su valor es su propia habilidad de producción (Cooper, 2015). De este modo, el contexto en el cual emerge el sujeto adolescente es el contexto neoliberal, en el cual el orden económico se integra en ámbitos en los cuales no solía ser una variable de análisis, como la familia, educación y Estado. El sujeto neoliberal se constituye como un sujeto que debe competir y rendir, buscando la adaptación a la lógica del mercado en todas las variables que constituyen su subjetividad, ya que mediante este rendimiento se constituye como sujeto reconocido.

La noción de sujeto emprendedor y neoliberalismo se puede relacionar con distintos conceptos desarrollados por la psicología positiva en la adolescencia. Un ejemplo son las investigaciones vinculadas al rol del optimismo en la salud y calidad de vida en adolescentes (Hamvai \& Pikó, 2010; Monzani, Steca, \& Greco, 2014), en donde las expectativas positivas propiciarían un mayor bienestar y mejores condiciones de salud en los adolescentes. Desde estos estudios, la emergencia del optimismo se explica como una cualidad psicológica, y por ende individual, en donde las variables ambientales (como familia, escuela y vecindarios) aparecen desde una lógica nuclear propia del neoliberalismo en donde lo social se reduce a lo interpersonal (Burman, 1993). Se refiere, por ejemplo, la importancia de que los adultos puedan promover la importancia del bienestar personal y el apoyo de los ambientes sociales (Thomson, Schonert-Reichl, \& Oberle, 2014), enfatizando de este modo lo interpersonal más que los elementos socioeconómicos, culturales 
o la misma participación de los jóvenes en torno a la construcción y promoción del bienestar.

Bajo la necesidad demandada desde el modelo político económico neoliberal de un sujeto emprendedor, es que emerge para una mejor integración la necesidad de competencias adecuadas para el emprendimiento y surgimiento del sujeto en el ámbito socioeconómico. El desarrollo económico exige una mayor y mejor preparación para su integración a la vida productiva, aplazándose este ingreso para generar un sujeto que responda con esta demanda mediante herramientas otorgadas desde el modelo como apropiadas para su inserción final (Ariés, 1973). La necesidad de esta adquisición de saberes y conocimientos hace que la necesidad de la escolarización sea obligatoria, así como de la formación superior, la cual cada vez más va dilatando el ingreso al mercado laboral de los sujetos, con lo cual la adolescencia emerge en este contexto político y económico particular. Tales prácticas y saberes que se reproducen en modelos actuales -como la psicología positiva-, vinculados a la causalidad, individualismo y a la "tiranía de la actitud positiva" (Prieto-Ursúa, 2006, p. 329), facilitan que los procesos de subjetivación se desarrollen desde la participación en la sociedad del rendimiento (Han, 2016). Un ejemplo son las denominadas estrategias de afrontamiento -como la resolución de problemas o el pensamiento positivo- propuestas por la Psicología Positiva, donde las investigaciones buscan incluso conocer las más adecuadas -referidas como activas- para que los adolescentes puedan alcanzar el bienestar y la autorregulación emocional (Pascual, Conejero, \& Etxebarria, 2016).

De este modo, las ideas propuestas por Foucault son el inicio para analizar cómo, a través de las transformaciones del modelo actual de la sociedad, los discursos socioeconómicos poseen una participación relevante en los modos de subjetivación de los individuos. Considerar el bienestar subjetivo y la felicidad como un factor relevante en la vida de los y las adolescentes no es una noción neutral, en tanto, la vida de los y las adolescentes hoy se encuentra atravesada por las prácticas propias del neoliberalismo y el empuje por el rendimiento, lo cual permea las prácticas cotidianas de los jóvenes (Ashman, Patterson, \& Brown, 2018).

Pero, discursos como el de la felicidad conllevan a construir la noción de bienestar desde la idea de libertad individual en una sociedad donde la idea de libertad se encuentra bajo las lógicas del rendimiento constante y la productividad, de la autovigilancia, y, como denomina Han (2013), del panóptico digital. La psicologización que el término felicidad tiene a su base, en tanto emoción, se puede relacionar con la identificación de la psique como recurso productivo actual en nuestra sociedad, mediante la búsqueda de la optimización de los procesos psicológicos individuales. Desde ahí, no es cualquier emoción la que podría favorecer esta optimización, siendo idóneo el énfasis hacia la positividad y felicidad: "la psicopolítica neoliberal es una política inteligente que busca agradar en lugar de someter" (Han, 2016, p.57).

Esto puede verse, por ejemplo, en los estudios vinculados a la felicidad y organizaciones, en donde " $(. .$.$) de alguna manera el capitalismo$ neoliberal 'ludifica' la vida y el trabajo para hacer de las interacciones sociales algo entretenido, incrementando así la productividad y la información disponible" (Baratas, 2017, p. 886). En este contexto, es posible describir el uso que se le da a estos discursos en las sociedades del rendimiento, vinculado al énfasis en la productividad y en la felicidad y bienestar subjetivo como estrategia para lograr el éxito en una sociedad donde el éxito ni la felicidad se definen neutralmente.

Para Seligman (2005), las personas felices tienen un mejor rendimiento cognitivo, menos enfermedades, son más productivos en el trabajo, las experiencias negativas les afectan menos y construyen mejores relaciones sociales. Así, estos discursos se transforman en

(...) un conjunto de prácticas que buscan conducir la vida conducta de los individuos, regular su campo de acciones, establecer un particular modo de relación del sujeto consigo mismo (...) y que son consistentes con ciertos objetivos y metas propios de la racionalidad política neoliberal. (De la Fabián \& Stetcher, 2013, p.31).

Así, la felicidad pasaría a ser un instrumento más de esta racionalidad.

Bajo estas condiciones, la sociedad actual en donde la psicología positiva se enmarca, requiere de una comprensión distinta en donde: "El enemigo ha dejado de ser el elemento extraño, frente al que hay que estar preparado mediante medidas 
preventivas, y ha pasado a ser el mismo sujeto el que está problematizado" (Cruz Ortiz de Landázuri, 2017 , pp. 196-197). Han (2012/2014) propone el pasaje desde la clave disciplinaria de la sociedad en la biopolítica, a la clave del rendimiento:

La sociedad del rendimiento está dominada en su totalidad por el verbo modal poder, en contraposición con la sociedad de la disciplina, que formula prohibiciones y utiliza el verbo deber (...). La llamada a la motivación, a la iniciativa, al proyecto, es más eficaz para la explotación que el látigo y el mandato. (Han, 2012/2014, p. 19).

Este nuevo lugar político y económico se manifiesta como un escenario particular para la construcción de la subjetividad adolescente. En el contexto neoliberal, emergen nuevas prácticas sociales y culturales, de la mano de la globalización. Pareciera ser que la adolescencia, sobre todo cuando muestra su alejamiento al bienestar subjetivo y la felicidad, se constituye como grupo que corporiza modos en que el discurso social tradicional es constantemente puesto en cuestión, develando contradicciones, acto que trasciende los rangos desarrollistas o etarios y muestra cómo la adolescencia se constituye también como una modalidad subjetiva particular, muchas veces en contradicción con los valores de las generaciones con las que convive (Le Breton, 2012).

Distintas problemáticas atribuidas a la adolescencia -muchas veces relacionada con la palabra crisis o periodo crítico (Erikson, 1974; Hihara, Umemura, \& Sugimura, 2019)- emergen en prácticas políticas y económicas que se posicionan desde el neoliberalismo, y que promueven condiciones que favorecen el malestar y sufrimiento en los sujetos, como la desigualdad, exclusión, individualización de las problemáticas, psicopatologización, empuje por el autocontrol, entre otras.

Así, buscar interpretar las dificultades y problemáticas vinculadas a la adolescencia a partir de la psicología positiva y bienestar psicológico, podría individualizar de manera ahistórica formas de manifestación de un malestar y sufrimiento que no es sólo subjetivo, sino también social. Le Breton (2012) plantea que hoy ser adolescente se hace más difícil debido a que la tarea de ser individuo es más compleja:
El individualismo contemporáneo implica, para el sujeto, el hecho de tener que definirse a través de sus propios referentes. Sin el sostén de las regulaciones colectivas, se ve obligado a tomar la iniciativa, a encontrar en sí mismo los recursos de sentido para seguir siendo actor de su existencia. (p. 30).

En un período de constante cuestionamiento identitario, dentro del cual se han perdido ritos y prácticas que den sentido al paso a un estatuto que deja atrás la potencialidad de la infancia, se refiere la problemática sobre el sentido y valor sobre este proceso, lo cual puede conllevar a las llamadas conductas de riesgo (Le Breton, 2012; Cancilliere, Yusufov, \& Weyandt, 2018), con las cuales las y las adolescentes buscarían encontrar un sentido al vacío subjetivo y simbólico que sienten, como malestar subjetivo y social que pasa a ponerse en acto por los y las adolescentes, debido a la falta de discursos sociosimbólicos que puedan sostener sus procesos de transformación identitaria.

Los nuevos lazos sociales en la adolescencia son movedizos, no implican el mero intercambio centralizado de capital cultural (político y económico), sino un alejamiento, división y puntos de referencia múltiples que se ofrecen al adolescente, con modalidades jerarquizadas y discursos que son reconocidos, versus discursos que buscarían ser disciplinarizados, en pos de la práctica del sujeto neoliberal. Sin embargo, la lógica de discliplinamiento transita hacia la transformación de estos discursos en su vertiente positiva, interpretándose desde las lógicas de las sociedades del rendimiento, a través, por ejemplo, del énfasis hacia un autocontrol de la psique, más que un control externo social hacia el cuerpo.

Un ejemplo es la investigación propuesta por Warren, Wray-Lake y Syvertsen (2018) titulada Becoming who they want to be: A cross-national examination of value-behavior concordance and mindfulness in adolescence en donde las nociones de rendimiento, autocontrol y coherencia se tornan centrales e incluso deseables para la adolescencia, relacionando estos elementos con la técnica del mindfulness, que corresponde a una interpretación occidental de la técnica de la meditación y que promueve la idea de la atención consciente en el presente, mediada por el propio control del individuo. Así, es el mismo sujeto adolescente el que pasa a ser problematizado, como individuo 
con características que deben mostrar coherencia y que podrían dificultar su bienestar subjetivo y felicidad. Al mismo tiempo, los y las adolescentes podrían -mediante técnicas individuales como el mindfulness- alcanzar por sí mismos este bienestar subjetivo y felicidad.

Por esto, en el contexto de las sociedades de rendimiento, es responsabilidad del mismo "sujeto emprendedor" su autorregulación y bienestar subjetivo, ya que él corporiza e individualiza la idea de una empresa, y sus bajas también están bajo su responsabilidad y agencia en la sociedad del rendimiento. Entonces, ¿cómo entender un bajo nivel de bienestar subjetivo y felicidad? Una posibilidad es comprender que este bajo nivel o que las problemáticas que podrían relacionarse a esta idea -como las conductas de riesgo o conflictos con las instituciones sociales- podrían representar más que una falla individual, un discurso subjetivo de malestar social.

De este modo, la clasificación de características que potencian el bienestar subjetivo y la felicidad no es tan simple como intenta establecerse. En base a esto, se hace necesario problematizar en torno no sólo a lo que implica la medición del bienestar sino a su vez a cómo se analizan los elementos que afectarían negativamente el bienestar. Al respecto, podría discutirse cómo la aparición de problemáticas en el bienestar subjetivo responde a una ruptura y una forma de expresión de malestar frente a los discursos propios del neoliberalismo. Es decir, pensar las problemáticas en el bienestar subjetivo y logro de la felicidad en la adolescencia no como individuales, sino también como modos de problematización y malestar frente al discurso adultocéntrico económico y político neoliberal en el cual el bienestar subjetivo parece inscribirse.

\section{EI bienestar subjetivo en la adolescencia: la mirada desde el malestar social}

A partir del análisis realizado, las problemáticas de bienestar subjetivo y felicidad en la adolescencia pueden entenderse no solo como problemáticas que aluden a lo individual o psicopatológico, sino también como modos de manifestar dificultades de acoplarse a la lógica de la felicidad desde lo individual, como expresión de un malestar frente al contexto social, económico y político en el cual se producen y transmiten los valores sociales bajo la lógica del mercado y neoliberalismo. Así, la adolescencia vista como conflicto o inadecuación podría remitir a la problematización de las condiciones del neoliberalismo, ya que la adolescencia como tal no deja de estar atravesada por variables contextuales que hacen a este grupo y modalidad subjetiva heterogénea (Lapointe, 2016).

De este modo, pareciera ser que la idea de la adolescencia como preparación o moratoria social (Erikson, 1974) ante la inserción en el mundo adulto dista de ser tan clara y esperada. Las instituciones tradicionales como la escuela, la familia o la religión cada vez son más puestos en cuestión y han develado su incapacidad para sostener en este momento subjetivo de los y las adolescentes y sus transformaciones, mayormente porque, a su vez, también se encuentran atravesadas por lógicas neoliberales.

Como menciona Foucault (1977a/2002), ya no se trata de instituciones restrictivas, sino más bien de instituciones de poder productivo, es decir de un poder sobre la vida, y mediante la regulación de la adolescencia, esta puede enfocarse a la satisfacción de las necesidades individuales y económicas, bajo las cuales se intenta mostrar un interés de protección que convive con lógicas económicas. Esto puede analizarse a partir, por ejemplo, de la educación, institución fuertemente relacionada con la adolescencia, considerada como un factor del bienestar subjetivo, y al mismo tiempo campo de producción económica en el contexto neoliberal.

La adolescencia, cuando es vista desde la incompletud, el conflicto y la exclusión, se muestra desde la psicopatologización, pero a su vez podría visibilizar las problemáticas sociales, en ocasiones diferenciándose de la visión estabilizadora del mundo social a partir de lógicas sociales, políticas y económicas. Un ejemplo es la representación respecto a la participación juvenil, la cual se tiende a relacionar con elementos vinculados a la disrupción. Sin embargo, esta diferenciación podría significar la expresión de un malestar respecto a estas lógicas, bajo lo cual el estudio del bienestar subjetivo y felicidad dista de ser un tema meramente individual y relacional, incluyendo elementos socioculturales y económicos en sus definiciones.

A través de expresiones corporales y conductuales -denominadas incluso ordálicas (Le Breton, 2012)-, o problemáticas en los indicadores 
de bienestar subjetivo, la hegemonía discursiva es puesta en cuestión, expresando un malestar social. La adolescencia ha sido históricamente hablada por el mundo adulto y, bajo esto, se ha expuesto como un terreno de intervención que vincula la metamorfosis corporal, la irrupción hormonal y el abandono de la infancia como modalidad de producción de una subjetividad que más que normarse debe además autocontrolarse: "El poder productivo desde la regulación de la vida se transforma en un poder de productividad desde la psique" (Cruz Ortiz de Landázuri, 2017: p. 199).

Por esto, las intervenciones más que prohibir, buscan transformar las acciones hacia la positividad amparada en discursos sobre todo científicos y psicologizantes, como un poder hacer (Han, 2014). Aún así, no se indaga en torno a cómo se enuncia cierta legitimidad sobre la felicidad y el bienestar, así como la relación que los individuos establecen con esta. Por otro lado, la operacionalización del bienestar subjetivo tampoco explora ciertos ideales de sujeto que establece el discurso de la psicología positiva.

La adolescencia fuera de la norma podría poner en cuestión estos discursos, corporizando la figura de la monstruosidad, como discurso adolescente, que pone en tensión la idea de cierto bienestar subjetivo que apela a un individuo que se agencia a sí mismo, mediante una discutible libertad. Así, la solución de los problemas pasa a ser puesta en la dimensión de la necesidad de un cambio individual, más que en el interés de escuchar que está detrás de estas manifestaciones, en tanto discursos que son producidos en contextos sociales específicos y aluden a un malestar respecto a las lógicas excluyentes, desiguales e individualizantes del discurso neoliberal, lo cual contribuye a la construcción subjetiva de este malestar.

\section{Adolescencia: del bienestar al monstruo político.}

La adolescencia pareciera visibilizar, en este tipo de expresiones que se distancian del bienestar subjetivo y felicidad, las contradicciones que la operacionalización respecto a la felicidad que el discurso de la psicología positiva transmite. El problema parece surgir cuando se vincula el bienestar subjetivo y la felicidad como hechos psicológicos individuales, homogéneos y ahistóricos, cuando más bien sus repercusiones van más allá de esta nomenclatura psicologizante, y se transforman en modos de producción de sujetos desde una gubernamentalidad específica:

(...) quizás ya no en absoluto, una enfermedad o un proceso patológico, sino cierto estado que se va a caracterizar como de desequilibrio, es decir, un estado en el cual los elementos funcionan de un modo que, sin ser patológico, sin ser portador de morbilidad, no es sin embargo un modo normal.(Foucault, 1975/2011, p. 284).

Pareciera que la adolescencia se transforma en ocasiones en un grupo que se muestra como fuera de parámetros estandarizables:

Lo que constituye a un monstruo humano en un monstruo no es simplemente la excepción en relación con la forma de la especie, es la conmoción que provoca en las regularidades jurídicas (ya se trate de las leyes matrimoniales, de los cánones del bautismo o de las reglas de sucesión). (Foucault, 1977b, p. 61).

La adolescencia fuera de los discursos del bienestar subjetivo y la felicidad construye una discursividad que manifiesta estar en el margen y fuera de la norma, deconstruyendo imágenes que persiguen el orden y la cohesión, para develar la fragmentación y desigualdad en la cual la realidad socioeconómica y política en la que el bienestar subjetivo se desarrolla. Pero tales características "anormales" pasan a ser responsabilidad del individuo adolescente, como refiere Foucault (1975/2011): “(...) esa especie de responsabilidad patológica del sujeto mismo con respecto a su propia enfermedad (...)" (p. 223), como si el sujeto emprendedor, también fuera responsable y empresario de sus fallas.

Estas manifestaciones fuera del bienestar subjetivo y la felicidad pueden pensarse a su vez como modos de problematizar la necesidad transmitida por la sociedad del rendimiento sobre el control de la propia psiquis con el fin de hacer para rendir bajo ideales propios de las lógicas neoliberales, como, por ejemplo, la autorregulación emocional para así alcanzar un mejor desarrollo cognitivo y social. Como plantean De la Fabián y Stecher (2013), una característica particular de la orientación de la psicología positiva es que sus individuos se caracterizan por no reflexionar sobre 
sí mismos ni mirar el pasado, focalizándose en la acción -el hacer- presente.

Todo lo que queda fuera de los indicadores del bienestar subjetivo parece corporizar la figura de un peligro, sobre todo para el propio individuo, ya que se vería afectada su felicidad. Para Negri (2007) el poder ha sido pensando como eugenésico, en tanto hay ciertas formas de vida que son reconocidas y bellas, mientras otras pasan a ser relegadas a la exclusión y explotación. La adolescencia en su vertiente ideal desde el discurso oficial de la psicología positiva puede ser pensada como una forma de vida que desea ser cuidada y protegida, debido a su potencial de desarrollo y capital, pero la adolescencia que se inscribe fuera de los marcos del bienestar pasa a ser excluida al plano de la monstruosidad, como características que deben ser transformadas desde el individuo:

La oposición monstruosa hace crecer al sujeto, vuelve epidémica su existencia y busca destruir al enemigo. No reconoce la ambigüedad, sino que la ataca, se enfrenta al límite y no diluye los márgenes, reconoce al otro sujeto como enemigo, y contra él, deviene potencia. (Negri, 2007, p. 104).

De este modo, los discursos de la Psicología Positiva enfatizan la psicologización y dan cuenta de la influencia del neoliberalismo en la comprensión de la salud y bienestar.

El monstruo político (Negri, 2007), problematiza tecnologías -como la psicología positiva y su conceptualización del bienestar subjetivo- que tienen por objetivo la construcción normativa de lo humano como ficción que contempla la realidad social y corporal, que termina siendo un collage identitario que adviene como mapa de poder e identidad política. Pareciera que el monstruo ha dejado de estar excluido, sino que se hace presente, se visibiliza -a partir de un bajo bienestar subjetivo, conductas de riesgos o movimientos sociales-, y frente a esto, los mecanismos de control político y económico -por ejemplo, el autocontrol o la estandarización de la norma del bienestar subjetivo- buscan aferrarlo.

A pesar de que el monstruo de igual forma penetra la realidad social, sí poseería un lugar en el cual se intentan poner en efecto prácticas de exclusión, de rechazo y de marginación. Algunas subjetividades no reconocidas presentan tensiones ante el discurso hegemónico y el ideal de bienestar subjetivo ofrecido como tarea del mismo sujeto, cuya normalización logra homogeneizarlos bajo un grupo que debe corregirse -o más bien autocorregirse- con el fin de alcanzar los indicadores esperados del bienestar subjetivo.

Exponerse como un campo de conflictos ante el neoliberalismo y sus políticas de bienestar implica a su vez la marginalidad en un modelo político, económico y social, en el cual advienen algunas subjetividades adolescentes, desde la exclusión social y la ausencia de reconocimiento por elementos sociales, económicos, de etnia y género, entre otros. La marginalidad es vista como un peligro y bajo esto, es importante realizar un análisis que discuta el abordaje respecto a la relación entre sociedad, control y adolescencia desarrollando perspectivas situadas en el contexto neoliberal actual, integrando la complejidad de la problemática mediante una discusión que visibilice esto. Un ejemplo es la noción de post panóptico, bajo el cual la extensión del control permea distintos espacios y se vincula con la sociedad de consumo, y busca problematizar y reinterpretar el modelo de sociedad desde la biopolítica a los escenarios neoliberales actuales (Castro, 2009).

Este espacio, en donde el llamado monstruo político emergería, se enfrenta a los modos de producción subjetiva actuales desde donde la psicología positiva y su concepto de bienestar subjetivo cobran sentido. Esto no es solo a nivel metodológico, destacando investigaciones de carácter cuantitativo y proponiendo el bienestar subjetivo y la felicidad como meta universal que debe ser evaluada para predecir y mediar en las problemáticas adolescentes; sino también a nivel teórico, ya que no problematiza la relación establecida entre bienestar subjetivo, felicidad y productividad en las sociedades actuales y sus implicancias en grupos particulares, como la adolescencia. La relación establecida entre bienestar subjetivo y cualidades vinculadas al rendimiento, como el mejor desarrollo cognitivo o la mejor productividad, da cuenta de la importancia de analizar los fundamentos que este concepto posee y las posibles relaciones de la psicología positiva con los discursos hegemónicos propios del neoliberalismo. Además, bajo esta lógica, un bajo índice de bienestar subjetivo y felicidad en la adolescencia podría interpretarse a su vez como un modo de problematizar estos 
discursos y visibilizar un malestar en torno a la sociedad neoliberal.

Se hace relevante reflexionar en torno a las implicancias políticas bajo las cuales distintos modelos psicológicos se posicionan. Dar cuenta de un discurso cientificista con el fin de validar prácticas y saberes, conlleva la naturalización y reproducción de discursos y lógicas neoliberales que se transmiten en grupos particulares, como los adolescentes. Estos discursos, amparados desde el método científico de investigación, favorecen la individualización y el énfasis en el rendimiento como discurso en un momento del desarrollo psicosocial en el cual los procesos de participación social, las tensiones con los discursos adultocéntricos y la construcción de discursos sociosimbólicos que signifiquen el pasaje desde la niñez y situarse desde otro lugar simbólico en la sociedad adulta es sumamente relevante.

Finalmente, es posible pensar que las características y conductas que se presentan en la adolescencia como alejadas del bienestar subjetivo y felicidad no corresponden sólo a manifestaciones individuales, sino también a modos de expresión respecto a un sufrimiento y malestar social sobre las condiciones de la sociedad neoliberal actual. Por esto, hablar de la anormalidad -en tanto lo que queda fuera de la norma del bienestar subjetivo y la felicidad- y la adolescencia implica pensar la norma no sólo desde lo restrictivo, sino también desde modos de deconstruir la individualización de la felicidad. Esto podría generar lineamientos de una nueva comprensión ante la adolescencia y a la subjetivación de este grupo desde la gubernamentalidad actual, a través de la vida no sólo como atrapada por la estandarización del bienestar ni como autocontrol, sino también desde la participación y el cuestionamiento respecto a la productividad como ideal que se instala en la adolescencia y en las sociedades, incluyendo elementos socioeconómicos, históricos, políticos y culturales en sus análisis. 


\section{Referencias}

Alfaro, J., Casas, F., \& López, V. (2015). Bienestar en la infancia y adolescencia. Psicoperspectivas. Individuo y Sociedad. 14 (1), 1-5. Recuperado de http://www.psicoperspectivas.cl/index.php/ psicoperspectivas/article/viewFile/601/409

Álvarez, Y. (2014). Relación del bienestar psicológico con los niveles de ansiedad: rasgo-estado en adolescentes. Revista de Ciencias Médicas La Habana, 20(1). Recuperado de http:// revcmhabana.sld.cu/index.php/rcmh/article/view/186/html

Antoine, P., Congard, A., Andreotti, E., Dauvier, B., Illy, J., \& Poinsot, R. (2018). A Mindfulness-Based Intervention: Differential Effects on Affective and Processual Evolution. Applied Psychology: Health and Well-being, 10(3), 368-390. doi: 10.1111/aphw.12137

Ariés, P. (1973). El niño y la vida familiar en el Antiguo Régimen. Madrid: Taurus.

Arps, E., Friesen, M., \& Overall, N. (2018). Promoting Youth Mental Health via Text-Messages: A New Zealand Feasibility Study. Applied Psychology: Health and Well-being, 10(3), 457480. doi: 10.1111/aphw.12143

Ashman, R., Patterson, A., \& Brown, S. (2018). 'Don't forget to like, share and subscribe': Digital autopreneurs in a neoliberal world'. Journal of Business Research, 92, 474-483. doi: https:// doi.org/10.1016/j.jbusres.2018.07.055

Baratas, M. (2016). Reseña del libro Psicopolítica. Neoliberalismo y nuevas técnicas de poder por Byung-Chul Han. Politica y Sociedad, 54(3). doi: 10.5209/POSO.55549

Bastaits, K., Pasteels, I., \& Mortelmans, D. (2018). How post-diverce paternal and maternal family trajectories relate to adolescents' subjective well-being? Journal of Adolescence, 64 , 98-108. doi: 10.1016/j.adolescence.2018.02.005

Bono, G., Froh, J., Disabato, D., Blalock, D., McKnight, P., \& Bausert, S. (2017). Gratitude's role in adolescent antisocial and prosocial behavior: A 4-year longitudinal investigation. The Journal of Positive Psychology, 1-3. doi: 10.1080/17439760.2017.1402078

Breuner C. \& Levine D. (2017). Committee on Adolescence. Adolescent and Young Adult Tattooing, Piercing, and Scarification. Pediatrics, 140(4). doi: 10.1542/peds.2017-3630

Burman, E. (1993). La Deconstrucción de la Psicología Evolutiva. Madrid: Aprendizaje Visor.

Casas, F., Fernández-Artamendi, S., Monserrat, C., Bravo, A., Bertrán, I., \& del Valle, J. (2013). El bienestar subjetivo en la adolescencia: Estudio comparativo de dos Comunidades Autónomas en España. Anales de Psicología, 29(1), 148-158. Recuperado de http://www.redalyc.org/Artículo.oa?id=16725574017

Castellá, J., Saforcada, E., Tonon, G., Rodriguez de la Vega, L., Mozobancyk, S., \& Bedin, L. (2012). Bienestar subjetivo de los adolescentes: un estudio comparativo entre Argentina y Brasil. Psychosocial Intervention, 21(3), 273-280. doi: 10.5093/in2012a24

Castro, R. (2009). La ciudad apestada. Neoliberalismo y postpanóptico. Rev. cienc. polít., 29(1), 165-183. doi: 10.4067/ S0718-090X2009000100009

Cloninger, R. \& García, D. (2015). The heritability and development of positive affect and emotionality. En M. Pluess (Ed.), Genetics of Psychological Well-Being: The role of heritability and genetics in Positive Psychology (pp. 97-113). New York: Oxford University Press.
Cooper, C. (2015). Entrepreneurs of the self: The development of management control since 1976. Accounting, Organizations and Society, 47, 14-24. doi: 10.1016/j.aos.2015.10.004

Cornejo, R. (2015). Temporalidad psíquica y subjetivación en la adolescencia. Rev. latinoam. psicopatol. fundam. 18(1), 62-73. doi: http://dx.doi.org/10.1590/1415-4714.2015v18n1p62.5

Christopher, J. \& Hickinbottom, S. (2008). Positive Psychology, Ethnocentrism, and the Disguised Ideology of Individualism. Theory \& Psychology, 18(5), 563-589. doi: 10.1177/0959354308093396

Cruz Ortiz de Landázuri, M. (2017). De la biopolítica a la psicopolítica en el pensamiento social de Byung-Chul Han. Athenea Digital, 17(1), 187-203. doi: 10.5565/rev/athenea.

Cuadra, H. \& Florenzano, R. (2003). El bienestar subjetivo: Hacia una Psicología Positiva. Revista de Psicología de la Universidad de Chile, XII(1), 83-96. Recuperado de www.revistapsicologia. uchile.cl/index.php/RDP/article/viewFile/17380/18144

De la Fabián, R. \& Stecher, A. (2013). Nuevos discursos acerca de la felicidad y gubernamentalidad neoliberal: "Ocúpate de ser feliz y todo lo demás vendrá por añadidura". Sociedad Hoy, 25, 29-46. Recuperado de http://www.redalyc.org/pdf/902/90239866003.pdf

Diener, E. (2000). Subjective well-being: The science of happiness and a proposal for a national index. American Psychologist, 55(1), 34-43. doi: 10.1037/0003-066X.55.1.34

Erikson, E. (1974). Identidad, juventud y crisis. Buenos Aires: Paidos

Eryilmaz, A. (2011). The Relationship Between Adolescents' Subjetive Well-being and Positive Expectations Towards Future. The Journal of Psychiatry and Neurological Sciences, 24, 209215. doi: 10.5350/DAJPN2011240306

Fairclough, N. (1989). Language and Power. London: Longman. Fairclough, N. (1992). Discourse and social change. Cambridge: Polity Press.

Fairclough, N. (1995). Critical Discourse Analysis. London: Longman

Favotto, L., Michaelson, V., \& Davison, C. (2017) Perceptions of the influence of computer-mediated communication on the health and well-being of early adolescents. International Journal of Qualitative Studies on Health and Well-being, 12(1), doi: 10.1080/17482631.2017.1335575

Fernández, L. (2008). Una revisión crítica de la psicología positiva: historia y concepto. Revista Colombiana de Psicología, 17, 161-176. Recuperado de https://revistas.unal.edu.co

Foucault, M. (1975/2011). Los Anormales (7ª Reimpresión). Buenos Aires: Fondo de Cultura Económica.

Foucault, M. (1977a/2002). Historia de la Sexualidad. La voluntad del saber ( $1^{\text {a }}$ Reimpresión). Buenos Aires: Siglo XXII Ediciones.

Foucault, M. (1977b). La vida de los hombres infames. La Plata: Editorial Altamira.

Foucault, M. (1979/2008). Nacimiento de la biopolítica $\left(1^{\mathrm{a}}\right.$ Reimpresión). Buenos Aires: Fondo de Cultura Económica.

García, M. (2002). El bienestar subjetivo. Revista Escritos de Psicología, 6, 18-39. Recuperado de https://dialnet.unirioja.es/ servlet/articulo?codigo $=281674$

Ghahari, S., Farhanghi, Z., \& Gheytarani, B. (2017). The effectiveness of teaching positive psychology on dysfunctional 
attitudes and emotional self-regulation of withdrawing addicts. European Psychiatry, 41, S410. doi: 10.1016/j.eurpsy.2017.01.345

Gillham, J., Adams-Deutsch, Z., Werner, J., Reivich, K., Coulter-Heindl, V., Linkins, M., Winder, B., Peterson, C., Park, N., Abenavoli, R., Contero, A., \& Seligman, M. (2011). Character strengths predict subjective well-being during adolescence. The Journal of Positive Psychology, 6(1), 31-44. doi: 10.1080/17439760.2010.536773

Gónzalez-Carrasco, M., Vaqué, C., Malo, S., Crous, G., Casas, F., \& Figuer, C. (2018). A Qualitative Longitudinal Study on the Well-being of Children and Adolescents. Child Indicators Research, doi: 10.1007/s12187-018-9534-7

Goodman, F., Disabato, D., Kashdan, T., \& Kauffman, S. (2017). Measuring well-being: A comparison of subjective well-being and PERMA. The Journal of Positive Psychology, 13(4), 321332. doi: 10.1080/17439760.2017.1388434

Hamvai, C. \& Pikó, B. (2010). "Is optimism good for your health?" The role of optimism in adolescent life and health. In Psychology of Optimism (pp. 123-143). Nova Science Publishers, Inc.

Han, B. (2012/2014). La agonía del eros. Barcelona: Herder.

Han, B. (2013). La sociedad de la transparencia. Barcelona: Herder.

\section{Han, B. (2016). Psicopolítica. Barcelona: Herder}

Hihara, S., Umemura, T., \& Sugimura, K. (2018). Considering the negatively formed identity: Relationships between negative identity and problematic psychosocial beliefs. Journal of Adolescence, 70, 24-32. doi: 10.1016/j.adolescence.2018.11.002

Janks, H. (1997). Critical Discourse Analysis as a Research Tool. Discourse: studies in the cultural politics of education, 18(3), 329-342. doi: 10.1080/0159630970180302

Khazaei, F., Khazaei, O., \& Ghanbari-H, B. (2017). Positive psychology interventions for internet addiction treatment. Computers in Human Behavior, 72, 304-311. doi: 10.1016/j. chb.2017.02.065

Lampropoulou, A. (2018). Personality, school, and family: What is their role in adolescents' subjective well-being. Journal of Adolescence, 67, 12-21. doi: 10.1016/j.adolescence.2018.05.013

Lapointe, A. (2016). Queering youth studies: Exploring the queer pedagogical insights in the Critical Youth Studies Reader. Journal of LGBT Youth, 13(3), 300-304. doi: 10.1080/19361653.2015.1089808

Le Breton, D. (2012). La edad solitaria. Adolescencia y sufrimiento. Santiago: LOM Ediciones.

Le Breton, D. (2014). Una breve historia de la adolescencia. Buenos Aires: Nueva Visión Argentina.

Lögdberg, U., Nilsson, B., \& Kostenius, C. (2018). "Thinking about the future, what's gonna happen?"- - How young people in Sweden who neither work nor study perceive life experiences in relation to health and well-being. International Journal of Qualitative Studies on Health and Well-being, 13(1). doi: 10.1080/17482631.2017.1422662

Lyngdoh, T., Liu, A., \& Srodhar, G. (2018). Applying positive psychology to selling behaviors: A moderated-mediation analysis integrating subjective well-being, coping and organizational identity. Journal of Business Research, 92, 142-153. doi: 10.1016/j.jbusres.2018.07.020

Miller, A. (2008). A Critique of Positive Psychology or 'The New Science of Happiness'. Journal of Philosophy of Education, 42(3-4), 591-608. doi: 10.1111/j.1467-9752.2008.00646.x
Monzani, D., Steca, P., \& Greco, A. (2014). Brief report: Assessing dispositional optimism in adolescence-factor structure and concurrent validity of the Life Orientation TestRevised. Journal of Adolescence, 37(2), 97-101. doi: 10.1016/j. adolescence.2013.11.006

Muha, T. \& Manion, J. (2010). Using Positive Psychology to Engage Your Staff During Difficult Times. Nurse Leader, 8(1), 50-54. doi: 10.1016/j.mnl.2009.12.002

Negri, A. (2007). El monstruo político. Vida desnuda y potencia. En G. Giorgi \& F. Rodríguez (Eds.), Ensayos sobre biopolítica. Excesos de vida (pp. 93-140). Buenos Aires: Paidos.

Ortuño-Sierra, J., Aritio-Solana, R., Chocarro de Luis, E., Navaridas Nalda, F., \& Fonseca-Pedrero, E. (2017). Subjective well-being in adolescence: New psychometric evidences on the satisfaction with life scale. European Journal of Developmental Psychology. doi: 10.1080/17405629.2017.1360179

Ouytsel, J., Ponnet, K., \& Walrave, M. (2017). The associations of adolescents' dating violence victimization, well-being and engagement in risk behaviors. Journal of Adolescence, 55, 66-71. doi: 10.1016/j.adolescence.2016.12.005

Pascual, A., Conejero, S., \& Etxebarria, I. (2016). Coping strategies and emotion regulation in adolescents: Adequacy and gender differences. Ansiedad y Estrés, 22(1), 1-4. doi: 10.1016/j. anyes.2016.04.002.

Prieto-Ursúa, M. (2006). Psicología Positiva: una moda polémica. Clínica y Salud, 17(3). Recuperado de http://scielo.isciii.es/pdf/ clinsa/v17n3/v17n3a07.pdf

Russell, E. \& Daniels, K. (2018). Measuring affective wellbeing at work using short-form scales: Implications for affective structures and participant instructions. Human Relations, 71(11), 1478-1507. doi: 10.1177/0018726717751034

Ryff, C. D. (2018). Well-Being With Soul: Science in Pursuit of Human Potential. Perspectives on Psychological Science, 13(2), 242-248. doi: 10.1177/1745691617699836

Sachs, K., Gillham, J., DeMaria, L., Andrew, G., Peabody, J., \& Leventhal, S. (2015). Building psychosocial assets and wellbeing among adolescent girls: A randomized controlled trial. Journal of Adolescence, 45, 284-295. doi: 10.1016/j. adolescence.2015.09.011

Seligman, M. \& Csikszentmihalyi, M. (2000). Positive Psychology: An introduction. American Psychologist, 55(1), 5-14. doi: 10.1037/0003-066X.55.1.5

Seligman, M. (2005). La auténtica felicidad. Bogotá: Imprelibros, S.A.

Seligman, M. (2011). Flourish: A new understanding of happiness, well-being-and how to achieve them. New York: Free Press

Swartz, M. (2018). The power of Positive Psychology. Journal of Pediatric Health Care, 32(6), 533. doi: 10.1016/j. pedhc.2018.09.001

Thomson, K., Schonert-Reichl, K., \& Oberle, E. (2014). Optimism in Early Adolescence: Relations to Individual Characteristics and Ecological Assets in Families, Schools, and Neighborhoods. Journal of Happiness Studies, 16(4), 889-913. doi: 10.1007/ s10902-014-9539-y

Tylka, T. L. (2012). Positive psychology perspectives on body image. In T. F. Cash (Ed.), Encyclopedia of body image and human appearance (pp. 657-663). San Diego, CA: Elsevier Academic Press. doi: 10.1016/B978-0-12-384925-0.00104-8 
Valle, J. (2013). Bienestar subjetivo en adolescentes: Un estudio comparativo entre dos comunidades autónomas. Revista Anales de la Psicología, 29(1), 148-158. doi: 10.6018/analesps.29.1.145471

Warren, M., Wray-Lake, L. \& Syvertsen, A. (2018) Becoming who they want to be: A cross-national examination of value-behavior concordance and mindfulness in adolescence. The Journal of Positive Psychology, 13(6), 605-616. doi: 10.1080/17439760.2017.1350741 